\title{
Editorial
}

Unfallchirurg 2015 · 118:574

DOI 10.1007/s00113-015-0034-7

Online publiziert: 2. Juli 2015

c) Springer-Verlag Berlin Heidelberg 2015
C. Krettek ${ }^{1} \cdot$ W. Mutschler ${ }^{2}$

${ }^{1}$ Unfallchirurgische Klinik, Medizinische Hochschule Hannover, Hannover, Deutschland

${ }^{2}$ Chirurgische Klinik und Poliklinik, Klinikum der LMU München, München, Deutschland

\section{Der Unfallchirurg ist offizielles Organ der Österreichischen Gesellschaft für Unfallchirurgie (ÖGU)}

Aufmerksame Leser werden es bemerkt haben: seit April diesen Jahres ist Der Unfallchirurg offizielles Organ der Österreichischen Gesellschaft für Unfallchirurgie mit seinen etwa 1700 Mitgliedern. Gerne sind wir diesem Anliegen unserer österreichischen Kolleg/innen gefolgt, denn der traditionell hohe Stellenwert der Unfallchirurgie im deutschsprachigen Raum ist maßgeblich von ihnen mitbegründet und -gestaltet worden. Wesentliche diagnostische und therapeutische, aber auch organisatorische und gesundheitspolitische Impulse kamen aus Österreich.

Unser Fach hat deshalb auch seit jeher vom bilateralen Austausch profitiert. Etliche Pioniere und Wegbereiter der Unfallchirurgie kamen aus Österreich (Böhler, Tscherne, Muhr u. a.) und haben das Fach in Deutschland geprägt und weiterentwickelt. Schüler dieser Persönlichkeiten wiederum gingen nach Österreich und belebten den Austausch.

Während z. B. das Wissen um die von L. Böhler erarbeiteten konservativen Therapieoptionen in Deutschland in den Hintergrund gerückt zu sein scheint, ist in Österreich dieses Wissen noch präsenter. So werden die Möglichkeiten für eine konservative Therapie proximaler Humerusfrakturen, -schaftfrakturen oder gar Berstungsfrakturen der Wirbelsäule in Österreich sehr viel deutlicher als Therapieoption wahrgenommen als bei uns. Das in Österreich noch vorhandene Wissen und Können um die konservative Therapie darf nicht verloren gehen und sollte auch uns zu Gute kommen. Gerne bietet Der Unfallchirurg eine Plattform dafür.
Es bestehen noch viele andere Gemeinsamkeiten. In Deutschland wird seit Langem an der Verbesserung der Polytraumaversorgung gearbeitet. In diesem Zusammenhang entstand das Traumaregister der DGU, an dem sich auch zahlreiche Kliniken aus dem Ausland beteiligen. Hier ist Österreich mit 18 von 37 ausländischen Kliniken führend.

Das in Deutschland in den letzten Jahren sehr erfolgreich aufgebaute Traumanetzwerk wird in Österreich mit großem Interesse wahrgenommen und in zwei als überregionale Traumazentren zertifizierten Kliniken gelebt.

Beider Länder Gesundheitssysteme zählen zu den teuren (aber auch besten) weltweit. Deutschland hat 2003 auf die ökonomische Problematik mit der Einführung der DRGs reagiert, was nun auch in Österreich initiiert wird. Unsere Zeitschrift beleuchtet diese ökonomisch und ethisch relevanten Themen seit Langem in den entsprechenden Rubriken und praxisrelevanten Artikeln.

Ebenfalls 2003 beschloss der Deutsche Ärztetag, die Musterweiterbildung zu ändern und die fast 100 Jahre bestehende Trennung zwischen Orthopädie und Unfallchirurgie durch Einführung des „Facharztes für Orthopädie und Unfallchirurgie" aufzuheben. Eine Entwicklung, die auch in Österreich zur Umsetzung ansteht.

Diese und viele andere Gemeinsamkeiten können in Zukunft noch besser kommuniziert werden mit Der Unfallchirurg als offiziellem Organ der Österreichischen Gesellschaft für Unfallchirurgie.
Wir freuen uns auf unsere Leser, Autoren, Beiräte und Reviewer aus Österreich.

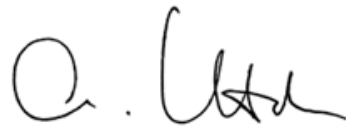

Prof. Dr. C. Krettek

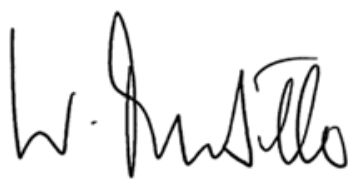

Prof. Dr. W. Mutschler
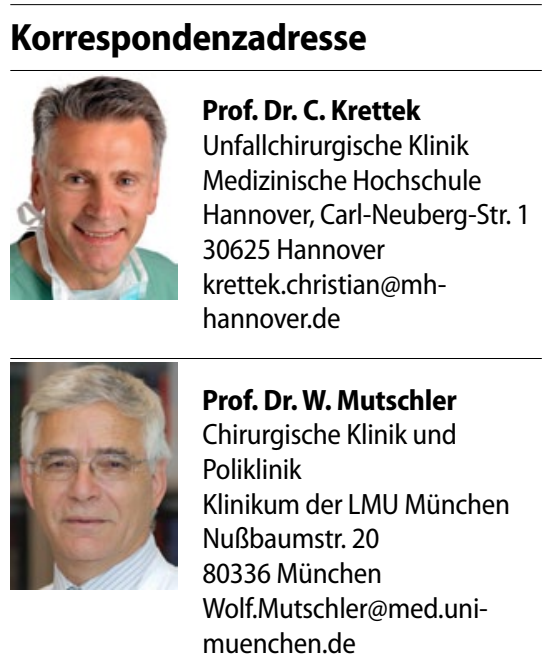

\section{Einhaltung ethischer Richtlinien}

Interessenkonflikt. C. Krettek und W. Mutschler geben an, dass kein Interessenkonflikt besteht. 
Hier steht eine Anzeige.

黛Springer 